\title{
ROTATIONAL ABSORPTION SPECTRUM OF HDO
}

M. W. P. STRANDBERG

TECHNICAL REPORT NO. 85

SEPTEMBER 25, 1948

RESEARCH LABORATORY OF ELECTRONICS

MASSACHUSETTS INSTITUTE OF TECHNOLOGY 
The research reported in this document was made possible through support extended the Massachusetts Institute of Technology, Research Laboratory of Electronics, jointly by the Army Signal Corps, the Navy Department (Office of Naval Research) and the Air Force (Air Materiel Command), under Signal Corps Contract No. W-36-039-sc-32037, Project No. 102B; Department of the Army Project No. 3-99-10-022. 


\title{
MASSACHUSETTS IMSTITUTE OF TECHIOLOGY
}

Research Laboratory of Electronics

Technical Report No. 85

September 25, 1948

ROTATIONAL ABSORPTION SPECTRUM OF HDO

M. W. P. Strandberg

\begin{abstract}
The $2_{2}-2_{I}, 3_{0}-3_{I}, 4_{-3}-3_{I}$, and the $5_{0}-5_{I}$ rotational absorption transitions of HDO have been observed and identified on the basis of their stark effect. By comparing the theoretical and experimental stark shift, the dipole moment for all the se transitions has been measured as $1.84 \pm .01$. The effective HOD angle 1 s also determined as $104^{\circ} \pm 30^{\prime}$ and the asymmetry coefficient, $K$, as about -.696 . The line breadth $\Delta v$ at one atmosphere is $.38 \pm .04 \mathrm{~cm}^{-1}$. None of these quantities is in disagreement with independent determinations.
\end{abstract}




\section{Introduction}

The intermediate heavy water molecule, HDO, has not been studied as fruitfully as have the $\mathrm{H}_{2} \mathrm{O}$ and $\mathrm{D}_{2} \mathrm{O}$ isotopic forms. The pure rotational spectrum of the intermediate molecule observed in the infrared is, of course, hopelessly complicated by absorption due to the inevitable $\mathrm{H}_{2} \mathrm{O}$ and $\mathrm{D}_{2} \mathrm{O}$ impurities. However, King, Hainer, and Cross ${ }^{I}$ (KHC) have re-analysed existing vibration-rotation data and on the basis of a rigid rotor model have published a table of expected absorption frequencles in the microwave region.

One might expect that little could be said about the HDO molecule if these absorption frequencies were measured since, for example, centrifugal distortion of the energy levels would obscure any relation between the absorption irequencies and the molecular structure. It is inevitable that with a molecule as complicated as HDO, an asymmetric top, those rotational energy levels having magnitudes of several hundred wave numbers may not be calculated exactly from measured energy-level differences of the order of one wave number.

On the other hand, such measurements are of value in determining the usefulness of a rigid rotor analysis, and in supplementing infrared data used in assigning term values. Stark effect measurements also provide additional structure data in the case of $\mathrm{HDO}$, as we shall show below. A study of the absorption due to $\mathrm{HDO}$ in the microwave region can thus be of use, although application of the data is certainly more limited than in the case of a linear or a symmetric top molecule.

\section{Experimental Measurements}

The sweep spectroscope and frequency standard used in these measurements have been described elsewhere. 2 since kHC have pointed out that centrifugal distortion effects cancel out rather well in $\Delta \mathrm{J}=0$ transitions, these transitions were first studied. The $5_{0}-5_{1}^{*}$ line at $22307.67 \mathrm{Mc} / \mathrm{sec}$ has been reported by Townes and Merritt, ${ }^{3}$ and by us. ${ }^{4}$ To obtain a more accurate analysis the stark effect for this transition was remeasured, and two other transitions, the $2_{1}-2_{2}$ and the $3_{0}-3_{1}$ were also measured at $10,278.99 \mathrm{Mc} / \mathrm{sec}$

1. G. W. KIng, R. M. Halner, and P. C. Cross, Phys. Rev. 71, 433 (1947).

2. M.W.P. Strandberg, T. Wentink, Jr., and R. L. Kyl, "Rotational Absorption Spectrum of OCS", RLE Technical Report No. 59, May 13, 1948.

3. C. H. Townes and F. R. Merritt, Phys. Rev. 70, 558 (I946).

4. Letter to Editor, Phys. Rev. 73 , 188 (1948).

* The J $\tau$ notation $w_{11}$ be used. J is the total angular momentum quantum number, and $\tau$ is a muning index over the energy levels within the given 'J group. 
and 50,236.90 $\mathrm{lc} / \mathrm{sec}$, respectively. The Stark measurements on these Iines enable positive identification to be made.

\section{Interpretation Results}

The theoretical Stark effect may be calculated for these transitions to compare with the observed perturbation. For perturbation energies ( $1 \cdot E$, where $\|$ is the electric dipole moment, $E$ is the electric field) much less than the energy difference between the asymmetric-top term values, the Stark effect is a second-order perturbation. The problem is one of calculating the quantum mechanical matrix elements of the direction cosines of the molecule-fixed axes to the space-fixed electric field axis. Induced polarization may generally be ignored as Goloen and Wilson ${ }^{l}$ have shown. Fortunately, necessary direction-cosine matrix elements have been tabulated by $\mathrm{CHK}^{2}$ in the form of line strengths, for various degrees of asymmetry of the molecule. They have also outlineda convenient method for calculating these matrix elements for any asymmetry. Some arithmetical juggling is necessary to recover the direction cosines themselves from the line strengths tabulated by CHK, since the line strengths are the sums of the squares of the directioncosine matrix elements over the magnetic quantum numbers and the three space directions. The term values in the presence of an electric field are given by conventional perturbation theory 3,4

$$
w_{J, \tau}=w_{J, \tau}^{(0)}+w_{J, \tau}^{(2)} \text { ergs }
$$

where $w_{J, \tau}^{(2)}$ is the second-order correction to the unperturbed energy $w_{J, \tau}^{(0)}$ and is calculated as

$$
w_{J, \tau}^{(2)}=E^{2} \quad \sum_{J^{\prime}, \tau^{\prime}}^{\prime} \frac{\mu_{g}^{2}\left|\Phi_{z, g}\left(J, \tau^{\prime}: J^{\prime} \tau^{\prime}\right)\right|^{2}}{w_{J, \tau^{\prime}}^{(0)}-w_{J^{\prime}, \tau^{\prime}}^{(0)}} \quad \mathrm{R} \text { ergs, }
$$

where

$$
\left|\Phi_{z, g}\left(J, \tau: J \cdot \gamma^{\prime}\right)\right|^{2}
$$

is the line strength for the transition $J, \tau \rightarrow J^{\prime}, \tau^{\prime}$ for a field along the space-fixed axis, $z$, interacting with a dipole moment along the molecule-fixed axis, g; this quantity is given in the CHK tables;

$$
\mu_{g}^{2}
$$
is the square of the component of the electric dipole moment along the molecule-fixed axis $g$;

1. S. Golden and E. B. W1lson, Jr. "Stark Effect for a Rigid Asymmetric Rotor", J. Chem. Phys. 16, 669 (1948).

2. P. C. Ćross, R. M. Haines and G. W. King, J. Chem. Phys. 12, 210 (1944).

3. Golden, Wlison, Jr., loc. c1t.

4. E. U. Condon and G. H. Shortley, "Theory of Atomic Spectra", Cambridge University Press, 1935. 


$$
R=\frac{M^{2}}{J^{*}\left(J^{*}+1\right)\left(2 J^{*}+1\right)}, \quad|M| \leq J^{*}=J \text { if } J=J^{\prime}
$$

or

$$
\begin{aligned}
& \mathrm{R}=\frac{\mathrm{J}^{*} \mathrm{~L}-\mathrm{M}^{2}}{\mathrm{~J}^{*}\left(4 \mathrm{~J}^{* 2}-\mathrm{I}\right)}, \quad|\mathrm{M}| \leq \mathrm{J}^{*}-1 \text { where } \mathrm{J}^{*}=\mathrm{J} \text { if } \mathrm{J}=\mathrm{J}^{\prime}+\mathrm{I} \\
& \text { or } J^{*}=J^{\prime} \text { if } J=J^{\prime}-I
\end{aligned}
$$

The primed summation indicates that the term $J^{\prime}, \tau^{\prime}=J, \tau$ is omitted.

The paper of Golden and Wilson gives an identical formulation of this solution in addition to a more general discussion of the problem for the case of degeneracy, a situation which has no bearing on the present problem.

The calculations themselves are straightforward. We have used three-point interpolation in the CHK tables for the line strengths. Threeplace logarithmic interpolation was used for cases where the line strength varied rapidiy with the asymmetry coefficient, $K$. ( $K$ is defined as $(2 b-a-c) /(a-c)$ where $a, b$, and $c$ are the maximum, intermediate, and minimum reciprocal moments of inertia).

Interpolation seemed unsatisfactory for the line strength of the $5_{0}-5_{1}$, and the $4_{-3}-3_{1}$ transitions, so we have calculated these two direction-cosine matrix elements exactly by using the method of CHK. ${ }^{2}$ KHC have investigated interpolation errors in their paper ${ }^{3}$ and the operation seems justifled in the other cases.

For HDO we have used $K=-.685,(a-c) / 2=8.495 \mathrm{~cm}^{-1}$ and $(a+c) / 2=14.88 \mathrm{~cm}^{-1}$, the latter two parameters being one-half the difference and the sum of the extreme reciprocal moments of HDO. These parameters were deduced from the KHC paper. The term value differences $w_{J, T}^{(0)}-w_{J, T}(0)$ must be calculated by using a rigld rotor model with the above parameters. In general the most important term is the one with the smallest energy difference in the denominator, and this usually corresponds to the transition frequency measured in the microwave region. Since this energy difference is therefore known very accurately, the major term in the above summation is thus known to an accuracy limited only by the validity of the interpolation of the line strength. The other terms essentially form a small correction factor so that rigid rotor term values are gufficlently accurate for use in their denominator.

1. Golden and W11son, loc. cit.

2. Cross, Haines and King, loc. c1.t.

3. King, Hainer, and Cross, loc. cit.

4. Ibid. 
Trouble was encountered in calculating the $3_{1}$ Stark perturbation for there are two near terms; one is the $3_{0}$, the other is the $4-3$ level. The $3_{0}-3_{1}$ energy denominator is 1mmediately calculated from the observed absorption frequency, but the $4_{-3}-3_{1}$ energy term is known rather inaccurately and is Iisted by $\mathrm{KHC}^{+}$as $1.13 \mathrm{~cm}^{-1}$, We therefore brought into the calculations at this point a frequency $v_{34}$ to stand for the expected $4_{-3}-3_{I}$ absorption Prequency, and carried the calculations through with this additional unknown. The other unknowns are the static dipole moment $\mu$, and the angle $\delta$ between the dipole moment and the molecule-fixed principal axis of the intermediate moment of inertia. The resulting stark shift in the $3_{0}-3_{1}$ absorption may then be given in the form

$$
\Delta v=\left(\frac{\mu E}{h c}\right)^{2} 10^{4}\left\{\sin ^{2} \delta\left(A+B M^{2}\right)+\cos ^{2} \delta\left(\frac{C}{v_{34}}+D+F M^{2}\right)\right\} \mathrm{Mc} / \mathrm{sec}
$$

where $A, B, C, D$, and $F$ are constants calculable by the procedure outined above, and $E$ is the electric fleld in kllovolts/om, h is Planck's constant and - Is the speed of Iight.

The observed stark shift was measured to have the form

$$
\Delta v=\left(0.577+0.087 \mathrm{M}^{2}\right) \mathrm{E}^{2} \mathrm{Mc} / \mathrm{sec} \text {. }
$$

If the predicted ratio of the $M$-dependent and $M$-independent term in $E q$. (2) is set equal to the observed ratio in Eq. ( 3 ), we obtain $v_{34_{2}}$ in terms of $A, B, C$, $D, F$, and $\delta$. The angle is known approximately as $20^{\circ} 30^{\prime}, 2$ and to this approximation a value Ior $v_{34}$ mas be calculated. This procedure was carried out roughly and gave a value of $20,000 \mathrm{Mc} / \mathrm{sec}$ or $.67 \mathrm{~cm}^{-1}$ for this frequency, instead of $34,000 \mathrm{Mc} / \mathrm{sec}$ or $1.13 \mathrm{~cm}^{-1}$ as predicted by the rigid rotor approximation. The only absorption in water mixtures which we have found in this region is one at $20,460.40 \mathrm{Mc} / \mathrm{sec}$.

It was then evident that the calculation should be carried out with $v_{34}$ taken as $20,460.40 \mathrm{Mc} / \mathrm{sec}$; and that the theoretical stark patterm for the $20,460.40 \mathrm{Mc} / \mathrm{sec}$ absorption should be computed on the basis of 1 ts being a $4_{-3}-3_{1}$ transition to check this identification with the observed Stark pattern.

The observed Stark splitting was so good even with an estimated value for $\delta$ that we assumed the $20,460.40 \mathrm{Mc} / \mathrm{sec}$ to be due to the $4_{-3}-3_{1}$ transition in HDO.

We mas now look at the complete plcture on the basis of this tentative identification to expose any inconsistencies. Since the rigid rotor predicted frequencies are so olose to the observed frequencies for $\Delta J=0$ transition, it may be assumed that centrifugal distortion in the two levels involved in each case is approximately the same. In any case, for a rigid rotor

1. King, Hainer, Cross, Ioc. c1t.

2. Ibid. 
approximation both levels must be described geometrically by the single parameter $\delta$, which we shall call $\delta \mathrm{J}$. Since we have four $J$ states involved, In the transitions, $J=2 \rightarrow 2,3 \rightarrow 3,3 \rightarrow 4$, and $5 \rightarrow 5$, there are only four values of $\delta_{r}$ which need be carried throughout the calculations. It must be remembered that from this point of view $\delta_{\mathrm{J}} 18$ essentially only a parameter which may or may not be related simply to a physical angle in the molecule, but it is the "effective" value of this angle for the transitions $J=2 \rightarrow 2,3 \rightarrow 3$, $3 \rightarrow 4,5 \rightarrow 5$, etc.

To be entirely consistent the direction-cosine matrix elements will vary with $\delta$, since they are a function of $K$ alone and it may be shown that in $\mathrm{HDO}$

$$
\mathrm{d} K \cong-.0043 \mathrm{~d} \delta_{\mathrm{J}}
$$

with $d \delta_{J}$ in degrees. For a variation of $\delta$ by one degree, $K$ will change by . 6 per cent and for all matrix elements except for the $4_{-3}-3_{1}$ term the corresponding change in the matrix elements will be no more than one-fifth of this, or about 0.1 per cent. Only the $3_{1}-4_{1}$ matrix element was therefore entered explicitly into the calculation as

$$
\left|\Phi_{\mathrm{zg}}\right|^{2}=(-.4954-.1158)\left(16-\mathrm{M}^{2}\right) \text {; }
$$

this expression $1 \mathrm{~s}$ in error by .3 per cent at $K=-.8$, and -.6 with no error at $K=-.7$.

The theoretical Stark effect may then be written in the Table I. The experimentally observed Stark shifts are also listed in Table $I$. By imposing the condion that the ratio of the $\mathrm{M}$-dependent to $M$-independent coefficients in both the observed and calculated stark formulae be equal, $\delta_{2}$ and $\delta_{5}$ may be determined uniquely. With values of $\delta$ so determined, the dipole moment may be obtained by using the condition that both the observed and calculated stark formulae have the same magnitude. The $\mu$ 's and $\delta$ 's $s 0$ determined are Iisted in Table II.

$$
\text { The } 3_{0}-3_{1} \text { and } 4_{-3}-3_{1} \text { transitions must be handled differently }
$$

since $k, \delta$, and $\mu$ enter explicitly into the Stark effect of these two transitions. The situation is under-determined, and so no explicit values for those parameters may be obtained. It would naturally be desirable to have $K_{J}$ and $\delta_{\mathrm{J}}$ for these two transitions turn out to be ldentical since the $3_{I}$ term participates in both. However, as we have indicated above, $\delta_{\mathrm{J}}$ must be considered only a parameter, or an effective angle in a rigid rotor stark-effect calculation. Also it would be well to have $\mu$ close to the value determined from the $J=2$ and $J=5$ transitions. Knowing the desired solutions then, one particular solution is: $\mu_{33}=1.85, \mu_{34}=1.83, \delta_{33}=19^{\circ} 50^{\prime}$, 


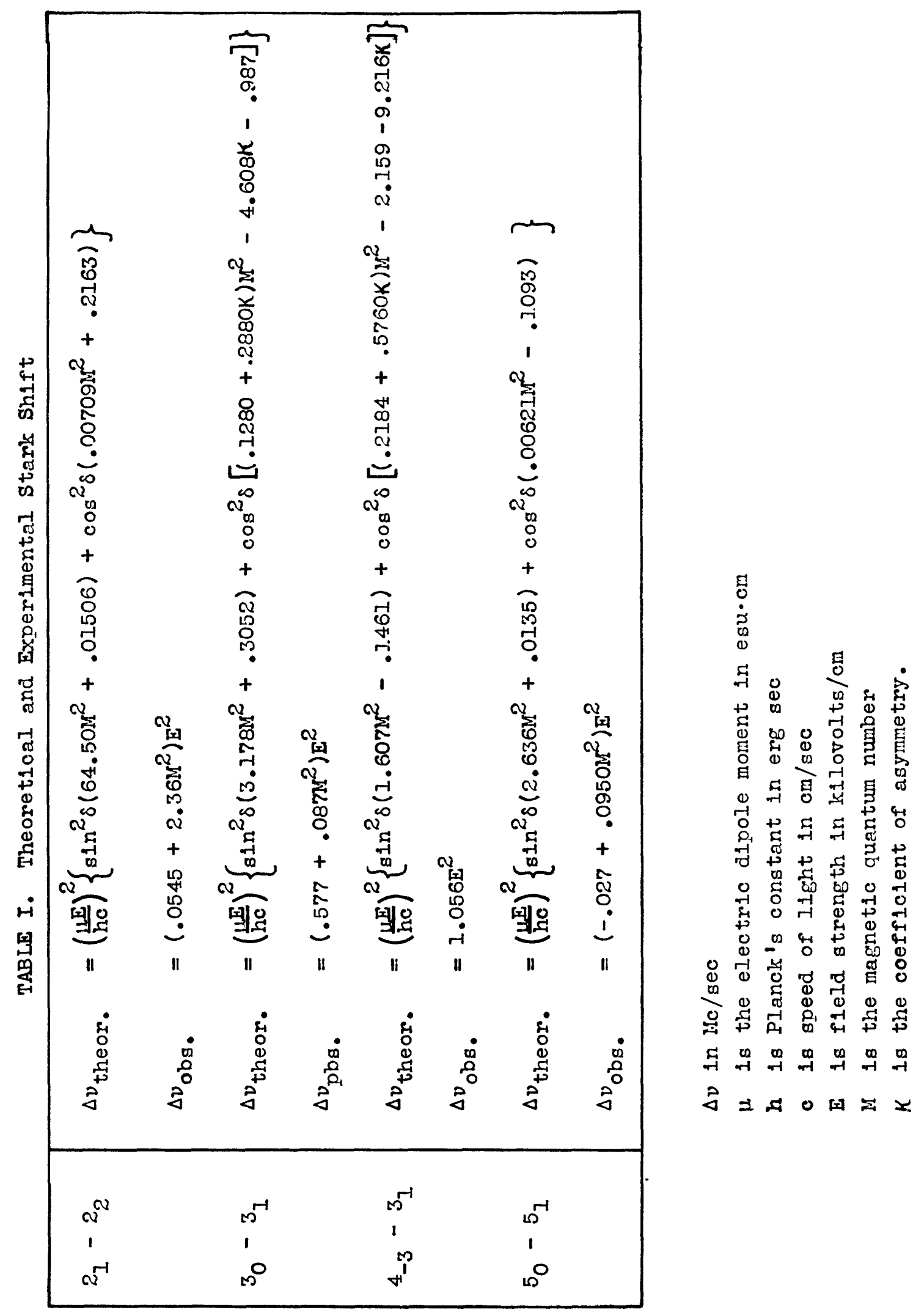


and $K=-.696$. This solution seems satisfactory from a physical point of view, and no further discussion of data reduction need be given. Incidental to the observation on the $3_{0}-3_{I}$ HDO absorption we have also measured the half-Iine breadth at half intensity, $\Delta v$, as $.010 \pm .001 \mathrm{~cm}^{-1}$ at $20 \mathrm{~mm}$ pressure. Thls extrapolates to a line breadth at one atmosphere of $.38 \pm .04 \mathrm{~cm}^{-1}$. Townes and Merritt have published a value of $.27 \pm .05 \mathrm{~cm}^{-\bar{I}}$ for the $50^{-} 5_{1}$ line breadth extrapolated to one atmosphere pressure. The two measurements agree rather well.

\begin{tabular}{|l|c|c|}
\hline \multicolumn{2}{|c|}{ TABLE II. Deduced Molecular Parameters } \\
\hline $2_{1}-2_{2}$ & $1.84 \pm 1 \%$ & $20^{\circ} 57^{\prime} \pm 1 \%$ \\
$3_{0}-3_{1}^{*}$ & 1.85 & $19^{\circ} 50^{\prime}$ \\
$4_{-3}-3_{1}^{*}$ & 1.83 & $19^{\circ} 50^{\prime}$ \\
$5_{0}-5_{1}$ & $1.83 \pm 2 \%$ & $20^{\circ} 44^{\prime} \pm 2 \%$ \\
\hline
\end{tabular}

Nor $K=-.696$

\section{Summary}

For four different transitions of low $\mathrm{J}$ the static dipole moment of $\mathrm{HDO}$ is seen to be a constant $1.84 \pm .01$. Centrifugal distortion makes no radical change in the electric moment between the $\mathrm{J}=2$ to $\mathrm{J}=5$ states. The angle between the moment of Inertia ellipsoid and the symmetry axis $\delta$ apparently undergoes no radical change, and may be determined as $20^{\circ} 30^{\prime} \pm 30^{\prime}$; the value of the HOD angle is then $104^{\circ} \pm 30^{\prime}$. The value of $\delta$ is in good agreement with the determination of this angle from the rigid rotor parameters as given by KHC. 2 Further, the asymmetry parameter may be shown to be about -.696 erom the Stark data. This value of $K$ is also in agreement with the KHC determination. It should be noted that though a value of $K$ had to be known before the stark data could be reduced, this value needed to be

1. Townes, and Merritt, loc. cit.

2. King, Hainer, and Cross, loc. c1t. 
known only roughly. It might be best to say that Stark effect.observations are capable of giving information on molecular structure which at least checks otherwise determined knowledge, and beyond that it gives information for a particular pair of rotational states instead of an average value over a number of rotational states. It may be seen from the above identification of the $4_{-3}-3_{1}$ transition that the stark effect is also a very useful tool to aid in assigning transition term values.

We have previously published similar observations on the Stark effect for the $5_{-1}-6_{-5}$ transition in $\mathrm{H}_{2} \mathrm{O} .2$ In this case the dipole moment proved to be about 5 per cent higher, than 1.84 debye, the measured result for HDO). A value of 1.84 debye is generally given for the dipole moment of $\mathrm{H}_{2} \mathrm{O}$ and $\mathrm{D}_{2} \mathrm{O}$ as measured from the temperature dependence of the polarization. It has been pointed out to us by E. B. Wilson, Jr. that the dipole moment is of interest to correlate experimental and theoretical studies of the line shape and intensity in this transition.

Van Vleck ${ }^{4}$ has shown that if a value of the dipole moment of 1.84 debye is used and the Iine breadth parameter is adjusted to fit the observed Iine shape, the calculated peak absorption for the $5_{-1}{ }^{-6} 6_{-5}$ absorption in $\mathrm{H}_{2} \mathrm{O}$ is low by about 20 per cent. If the moment observed in microwave Stark measurement for this transition is used, the discrepancy is reduced to 10 per cent and the agreement of theory with experiment is markedly enhanced. Van vleck mentions other effects which could also contribute to this discrepancy, so that for the present one can only say that these absorption studies are not in disagreement with the high dipole moment determined from the stark effect.

\section{Acknowledgment}

The author is grateful for the assistance of J. G. Ingersoll, T. Wentink and R. Hiliger in taking the experimental data upon which this paper depends.

1. For sim1lar use of the stark effect for transition laentiflcation in SO2, see B. P. Dalley, S. Golden, and E. B. Wilson, Jr. Phys. Rev. 72, 871 (1947). Previously the author has suggested, Bull. Amer. Phys. Soc. Paper YI: Phys. Rev. 74, 1245 (1948), that the $20,460.40 \mathrm{Mc} / \mathrm{sec}$ absorption might be due to $\mathrm{D} 2 \mathrm{O}$. The argument, though not incorrect is not so conclusive as the interlocking evidence presented here to assign it to the 4-3 - 31 transition in HDO.

2. S. Golden, T. Wentink, R. HIllger, M. W. P. Strandberg, Phys. Rev. 73, $92(1948)$.

3. See, for instance, Technical Report II, "Tables of Dipole Moments", Laboratory for Insulation Research, M.I.T.

4. J. H. Van vieck, Phys. Rev. 7l, 425 (i947). 